\title{
Inventário Florestal Utilizando Técnicas de Silvicultura de Precisão em Povoamentos de Eucalyptus grandis Hill ex Maiden
}

\author{
Mauricio Castro dos Santos ${ }^{1}$, Marcelo Roveda ${ }^{2}$, Magda Lea Bolzan Zanon ${ }^{3}$, \\ Afonso Figueiredo Filho², Mailson Roik², João Maurício Pacheco ${ }^{4}$, \\ Vanessa Scavinski ${ }^{4}$ \\ ${ }^{1}$ Florescer Serviços Ambientais, Frederico Westphalen/RS, Brasil \\ ${ }^{2}$ Universidade Federal do Paraná - UFPR, Curitiba/PR, Brasil \\ ${ }^{3}$ Universidade Federal de Santa Maria - UFSM, Frederico Westphalen/RS, Brasil \\ ${ }^{4}$ Universidade Estadual do Centro Oeste - UNICENTRO, Irati/PR, Brasil
}

\begin{abstract}
RESUMO
Este estudo teve por objetivo determinar o estoque de madeira por amostragem sistemática e pelas tecnologias de geoinformação a fim de elaborar mapas de produtividade florestal, como forma de pós-estratificação do inventário florestal. Foram alocadas 24 parcelas de $625 \mathrm{~m}^{2}$ posicionadas a cada $70 \mathrm{~m}$ de distância. $\mathrm{O}$ mapa de produtividade foi gerado após seleção de um modelo para ajuste do semivariograma entre os volumes das parcelas. Estimou-se um volume total de $3.753,9 \mathrm{~m}^{3}$ com o inventário florestal por amostragem sistemática, enquanto com o mapa de produtividade elaborado com técnicas de silvicultura de precisão determinou-se um volume total de $3.768,9 \mathrm{~m}^{3}$. A análise de variância comprovou que existe diferença significativa entre as médias dos estratos e a partir desta constatação as estimativas foram geradas por estrato, estimando-se então, um volume total de $3.772,9 \mathrm{~m}^{3}$. A técnica de silvicultura de precisão mostrou ser uma ferramenta eficiente na pós-estratificação de inventários florestais.
\end{abstract}

Palavras-chave: variabilidade espacial, geoestatística, produtividade florestal.

\section{Forest Inventory Using Precision Forestry Techniques in Eucalyptus grandis Hill ex Maiden Stands}

\begin{abstract}
This study aimed to determine the wood stock through systematic sampling and geoinformation technologies in order to elaborate maps of forest productivity as a forest post-stratification inventory. We positioned 24 plots of $625 \mathrm{~m}^{2}$ each ina distance of $70 \mathrm{~m}$ from each other. The yield map was generated after selecting a model for semivariogram adjustment between volumes in the plots. A $3,753.9 \mathrm{~m}^{3}$ total volume was estimated in the forest inventory through systematic sampling, while the yield map produced with precision forestry techniques showed a total volume of $3,768.9 \mathrm{~m}^{3}$. The analysis of variance showed that there is a significant difference between the means of the strata and based on this fact, the estimates were generated per stratum, then the total volume of $3,772.9 \mathrm{~m}^{3}$ was estimated. The precision forestry technique proved to be an efficient tool in the post-stratification of forest inventories.
\end{abstract}

Keywords: spatial variability, geoestatistical, forest productivity. 


\section{INTRODUÇÃO}

Os recursos florestais no Brasil vêm sendo explorados desde o início da colonização do país. Fatores como o crescimento da população e o desenvolvimento de novas tecnologias de utilização da madeira, aliados ao aumento do comércio e circulação desse produto, e a constante busca por novas áreas favoráveis às atividades agrícola e pecuária fizeram com que as florestas sofressem mudanças na sua estrutura original. Em decorrência disso, faz-se necessário aumentar e melhorar a produção de florestas plantadas para garantir a disponibilidade dessa matéria-prima consumida e a redução dos impactos sobre os ambientes nativos (Hack, 2007).

Segundo Lima et al. (2010), o gênero Eucalyptus é a essência florestal que mais atende as necessidades de reposição de matéria-prima para a fabricação de papel no Brasil, com crescimento médio de $45 \mathrm{~m}^{3} \cdot \mathrm{ha}^{-1}$.ano ${ }^{-1}$ de madeira. Dentre as diferentes espécies promissoras na formação de povoamentos plantados, esse gênero compreende um grande número de espécies e, consequentemente, uma alta variabilidade no que diz respeito às características físicas, mecânicas e estéticas, possibilitando a substituição de várias espécies nativas (Marchiori \& Sobral, 1997).

Péllico \& Brena (1997) comentam que em alguns inventários florestais o objetivo é simplesmente estimar o volume total de uma espécie, com isso o inventário florestal se entende como um instrumento informativo do volume de madeira. Porém, com o avanço da tecnologia, buscam-se informações com maior nível de detalhes sobre as áreas florestais, a fim de definir zonas específicas de manejo.

O manejador florestal normalmente obtém um valor médio de produtividade e incremento. Apesar de toda a variabilidade espacial ser detectada, dificilmente a mesma é espacializada na área de estudo. Desse modelo faz-se necessário o pleno conhecimento e quantificação da variabilidade da produtividade em florestas.

A silvicultura de precisão baseia-se na coleta e análise de dados geoespaciais localizadas nas florestas, com a exatidão e a precisão adequadas (Vettorazzi \& Ferraz, 2000), com potencial para uma nova forma de administração das florestas. A inclusão das variáveis espaciais e temporais dos fatores de produção e a incorporação de tecnologias como os sistemas de informações geográficas (SIG), sensoriamento remoto (SR), sistemas de posicionamento global (GPS) auxiliam e permitem intervenções precisas no povoamento (Brandelero et al., 2007).

$\mathrm{Na}$ análise da silvicultura de precisão destaca-se a geoestatística, que analisa a dependência espacial de dados georreferenciados aos quais é ajustado o semivariograma, representado pelo gráfico da semivariância em função das distâncias entre observações (Vieira, 2000). A partir do semivariograma pode-se confeccionar o mapa de krigagem para cada atributo pesquisado, do solo ou da planta, os quais representam a variabilidade espacial dos dados (Carvalho et al., 2012).

A utilização do SIG e da silvicultura de precisão possibilitam a criação de conjuntos de mapas que servem como instrumentos de decisão para o profissional florestal, munindo-o, dessa forma, com conhecimento sobre a distribuição dos atributos pesquisados. Trabalhos utilizando essa técnica em florestas foram elaborados por Höck et al. (1993), Biondi et al. (1994), Nanos et al. (2001), Rufino et al. (2006), Kanegae et al. (2006), Brandelero et al. (2007), Bognola et al. (2008), Mello et al. (2009), Leal et al. (2011), Rosa et al. (2011), Santos et al. (2011), Gonçalves et al. (2012), Carvalho et al. (2012), Guedes et al. (2012) e Pelissari et al. (2014), entre outros.

Segundo Péllico \& Brena (1997), a pós-estratificação é a divisão de estratos efetuada após a coleta de dados, podendo ser identificadas sua variabilidade e delimitação. Segundo os mesmos autores, a análise de variância (ANOVA) identifica se existe diferença significativa entre as médias dos estratos. Caso ocorra diferença, a amostragem estratificada trará vantagens no que se refere a custo e precisão do inventário.

Dessa forma, visando contribuir para o desenvolvimento desse enfoque nos inventários florestais, este trabalho objetivou determinar o estoque de madeira em um povoamento de Eucalyptus grandis Hill ex Maiden e avaliar a potencialidade da elaboração de mapas de produtividade florestal com o uso da geoestatística como forma de pós-estratificação em um inventário florestal. 


\section{MATERIAL E MÉTODOS}

\subsection{Caracterização da área de estudo}

Povoamento de E. grandis proveniente de clones localizado no município de Derrubadas, estado do Rio Grande do Sul, entre as coordenadas $27^{\circ} 26^{\prime} 40^{\prime \prime}$ e $27^{\circ} 26^{\prime} 60^{\prime \prime}$ de latitude sul e $50^{\circ} 81^{\prime} 40^{\prime \prime}$ e $50^{\circ} 81^{\prime} 54^{\prime \prime}$ de longitude oeste, em uma área de 12,79 hectares (ha). O plantio tem 6,5 anos de idade, primeira rotação e espaçamento $2,5 \mathrm{~m} \times 3 \mathrm{~m}$ (1.333,3 plantas/hectare). Para o preparo do solo foi utilizado o conjunto trator e subsolador, com profundidade de $40 \mathrm{~cm}$ na linha de sulco.

Segundo a classificação de Köppen, o município se enquadra no tipo climático Cfa (Moreno, 1961), com solos denominados Cambissolo eutrófico e distrófico $\mathrm{Ta}$ e Tb Achernozêmico e moderado, textura argilosa, fase pedregosa, com solos Litólicos eutróficos (IBGE, 1986).

\subsection{Medição nas unidades de amostra}

Nas unidades de amostra de $625 \mathrm{~m}^{2}(25 \mathrm{~m} \times 25 \mathrm{~m})$ foram mensurados o diâmetro a $1,30 \mathrm{~m}$ do solo (DAP) de todos os indivíduos, com auxílio da fita métrica, e a altura total das 15 árvores iniciais e das seis mais grossas, com hipsômetro Vertex VI. O modelo usado para estimar a altura total ( $h$ ) das árvores foi escolhido após o ajuste de 10 equações, sendo a equação log $(\mathrm{h}-1,30)=1,61891-4,40098 \mathrm{~d}^{-1}(\mathrm{~h}=$ altura total em metros; $\mathrm{d}=$ diâmetro a $1,30 \mathrm{~m}$ do solo em centímetros; $\ln =$ logaritmo neperiano; $\mathrm{e} \varepsilon \mathrm{i}=$ erro aleatório) escolhida por apresentar as melhores estatísticas, com coeficiente de determinação ajustado $\left(\mathrm{R}_{\text {Ajust }}^{2}\right)$ de 0,58 e erro padrão da estimativa $\left(\mathrm{S}_{\mathrm{yx}}\right)$ de $6 \%$. Os resultados da relação hipsométrica corroboram trabalho de Sousa et al. (2013) para povoamentos de Eucalyptus urophylla conduzido em regime de alto fuste e talhadia, no estado da Bahia, e Jesus et al. (2015), em povoamento clonal de Eucalyptus urophylla $\times$ Eucalyptus grandis, no Distrito Federal.

\subsection{Inventário florestal tradicional}

Estruturou-se o inventário florestal tradicional, criando-se uma malha de amostragem sistemática com intervalos (K) de $70 \mathrm{~m}$ entre parcelas de 0,5 ha do grid da área de 12,79 ha, totalizando 24 parcelas amostrais com as respectivas coordenadas centrais. $\mathrm{O}$ volume das árvores das unidades amostrais foi estimado da seguinte forma: $\mathrm{v}=$ g.h.f, em que: $\mathrm{v}=$ volume total com casca da árvore $\left(\mathrm{m}^{3}\right) ; \mathrm{g}=$ área transversal com casca a $1,3 \mathrm{~m}\left(\mathrm{~m}^{2}\right) ; \mathrm{h}=$ altura total $(\mathrm{m}) ; \mathrm{f}=$ fator de forma, sendo considerado um valor médio de 0,438, conforme proposto por (Finger, 1997). Nas análises do inventário florestal convencional utilizou-se o processo de amostragem sistemático e admitiu-se um erro máximo de $10 \%$ a um nível de probabilidade de confiança igual a $95 \%$.

\subsection{Análise geoestatística}

Para aplicar as técnicas de silvicultura de precisão, utilizou-se a malha de amostragem do inventário florestal. Os pontos da malha foram transferidos para o GPS de navegação HC 60, onde se procedeu às demarcações das parcelas e respectivas medições dos parâmetros requeridos pelo inventário.

Para o inventário com técnicas de silvicultura de precisão foi utilizada a análise geoestatística, para identificação da possível dependência espacial entre as unidades de amostra, por meio da determinação das semivariâncias (Equação 1), considerando o posicionamento geográfico das unidades amostrais no campo e o posterior cômputo das distâncias (h) e das diferenças numéricas da variável $(Z)$ na malha de pontos.

$$
\gamma(\mathrm{h})=\frac{1}{2 \mathrm{~N}(\mathrm{~h})} \sum_{\mathrm{i}=1}^{\mathrm{N}(\mathrm{h})}\left\{\left[\mathrm{Z}\left(\mathrm{x}_{\mathrm{i}}+\mathrm{h}\right)-\mathrm{Z}\left(\mathrm{x}_{\mathrm{i}}\right)\right]^{2}\right\}
$$

em que: $\gamma(\mathrm{h})=$ semivariância da variável $\mathrm{Z}\left(\mathrm{x}_{\mathrm{i}}\right)$; $\mathrm{h}=$ distância $(\mathrm{m})$; e $\mathrm{N}(\mathrm{h})=$ número de pares de pontos medidos $Z(x i)$ e Z(xi+h), separados por uma distância $h$.

As semivariâncias foram determinadas entre os pontos das unidades amostrais equidistantes, com a regularização da malha amostral por meio de uma tolerância angular de $22,5^{\circ}$ e passo de 70 metros. Esse processo foi repetido em quatro direções no plano espacial, $0^{\circ}(\mathrm{S}-\mathrm{N}) ; 45^{\circ}$ (SO-NE); $90^{\circ}(\mathrm{O}-\mathrm{L})$; e $135^{\circ}$ (NO-SE), das quais foi obtida a matriz das semivariâncias médias entre as distâncias equivalentes, além da quantificação dos pares de unidades amostrais computadas, conforme descrito em Pelissari et al. (2014).

Os modelos de semivariogramas teóricos utilizados para descrever a estrutura da dependência espacial foram 
o esférico (Equação 2), o exponencial (Equação 3) e o gaussiano (Equação 4), utilizando-se o programa $\mathrm{GS}^{+}$.

$\gamma(h)= \begin{cases}C_{0}+C\left[\left(\frac{3}{2}\right)\left(\frac{h}{A}\right)-\left(\frac{1}{2}\right)\left(\frac{h}{A}\right)^{3}\right] & \text { para } h \leq A \\ C_{0}+C & \text { para } h>A\end{cases}$

$\gamma(h)=C_{0}+C\left(1-e^{-h / A}\right)$

$\gamma(h)=C_{0}+C\left(1-e^{-h^{2} / A^{2}}\right)$

em que: $\gamma(\mathrm{h})=$ semivariância da variável $\mathrm{Z}\left(\mathrm{x}_{\mathrm{i}}\right)$; $\mathrm{h}=$ distância $(\mathrm{m}) ; \mathrm{C}_{0}=$ efeito pepita; $\mathrm{C}=$ variância $\mathrm{a}$ priori; $\mathrm{C}_{0}+\mathrm{C}=$ patamar; e $\mathrm{A}=$ alcance $(\mathrm{m})$.

O efeito pepita $\left(\mathrm{C}_{0}\right)$ é o valor da semivariância para a distância zero e representa o componente da variação ao acaso; o patamar $\left(\mathrm{C}_{0}+\mathrm{C}_{1}\right)$ é o valor da semivariância em que a curva estabiliza sobre um valor constante, aproximadamente igual à variância dos dados; a variância a priori $\left(\mathrm{C}_{1}\right)$ é dada pela diferença entre o patamar e o efeito pepita; e o alcance (A) é a distância da origem até onde o patamar atinge valores estáveis, expressando a distância além da qual as unidades de amostra não são correlacionadas (Trangmar et al., 1985).

O ajuste dos semivariogramas teóricos foi efetuado pelo Método dos Quadrados Mínimos Ponderados (Weight Least Squares - WLS). Na análise espacial, a avaliação e a seleção dos modelos nos ajustes dos semivariogramas teóricos foram realizadas com base na menor soma de quadrados dos desvios ponderados (SQDP) e maior coeficiente de determinação $\left(\mathrm{R}^{2}\right)$ e na validação cruzada, a qual compara o valor observado ao valor predito na unidade de amostra. Os valores de $\mathrm{R}^{2}$ variam de 0 a 1 e quanto mais próximo de 1 , mais eficiente é o modelo para representar o fenômeno estudado (Isaaks \& Srivastava, 1989).

Para analisar o grau de dependência espacial (GD) das variáveis em estudo utilizou-se a classificação de Cambardella et al. (1994), em que foram considerados com dependência espacial forte os semivariogramas com efeito pepita menor ou igual a $25 \%$ do patamar; com dependência moderada quando o GD ficou entre $25 \%$ e $75 \%$; e com dependência fraca quando o GD foi maior que $75 \%$.

A interpolação e espacialização do volume total foram realizadas por meio da krigagem ordinária pontual, sendo elaborado um projeto de silvicultura de precisão no software CR Campeiro 7. A metodologia de elaboração do projeto é apresentada em Giotto (2008).

\subsection{Inventário tradicional com pós-estratificação}

Após a elaboração do mapa de produtividade e definição das classes de volume, realizou-se a Pós-Estratificação do Inventário Tradicional, efetuando-se Análise de Variância da Estratificação (ANOVA) a fim de verificar a necessidade ou não da pós-estratificação dos dados de volume das parcelas amostradas. A ANOVA verifica a existência ou não de diferença significativa entre as médias dos estratos, e caso ocorra diferença entre as médias, a amostragem estratificada trará vantagens no que se refere a precisão e custo do inventário (Péllico \& Brena, 1997).

\section{RESULTADOS E DISCUSSÃO}

\subsection{Inventário florestal tradicional}

As principais estimativas obtidas no inventário florestal tradicional são apresentadas na Tabela 1 . O volume médio e a área basal por hectare apresentaram valores de $293,5 \mathrm{~m}^{3} /$ ha e $24,6 \mathrm{~m}^{2} /$ ha, respectivamente, ocasionando um erro de amostragem de 5,3\%. Após efetuarem-se os cálculos necessários foi observado que o número de parcelas foi suficiente para representar os valores quantitativos volumétricos.

\subsection{Análise geoestatística}

Com o efeito pepita $\left(\mathrm{C}_{\mathrm{o}}\right)$ foram observados valores inferiores a 460,5 (Tabela 2) para o volume; a variância dos dados foi mais elevada para o modelo gaussiano,

Tabela 1. Resultados do inventário florestal pelo processo de amostragem sistemática.

Table 1. Results of forest inventory through the systematic sampling process.

\begin{tabular}{|c|c|}
\hline Diâmetro médio $(\mathrm{cm})$ & 21,4 \\
\hline Altura média $(\mathrm{m})$ & 26,8 \\
\hline Altura dominante média $(\mathrm{m})$ & 28,9 \\
\hline Área basal média $\left(\mathrm{m}^{2} / \mathrm{ha}\right)$ & 24,6 \\
\hline $\begin{array}{l}\text { Volume total com casca médio } \\
\text { em pé }\left(\mathrm{m}^{3} / \mathrm{ha}\right)\end{array}$ & 293,5 \\
\hline Erro relativo (\%) & 5,3 \\
\hline Intervalo de confiança $\left(\mathrm{m}^{3} / \mathrm{ha}\right)$ & $278,1 \leq \bar{x} \leq 308,9$ \\
\hline $\begin{array}{l}\text { Volume total em pé } \\
\left(\mathrm{m}^{3} / 12,79 \mathrm{ha}\right)\end{array}$ & $3.753,9$ \\
\hline
\end{tabular}


variância ocasionada por erros não identificados pela técnica (Vieira, 2000). A dependência espacial do volume em povoamentos do gênero Eucalyptus também foi verificada por Mello et al. (2005, 2006), Kanegae et al. (2006) e Guedes et al. (2012), entre outros.

Os alcances (A) apresentaram variação entre os modelos testados de 384,3 a 441,0 (Tabela 2), sendo indicadores da distância limite em que há correlação entre pares de unidades amostrais no espaço (Vieira, 2000). Mello et al. (2005) utilizaram o modelo exponencial para estudo da dependência espacial do volume em Eucalyptus grandis na região sul do estado de São Paulo e observaram alcance prático de 700 metros.

Pode-se observar forte dependência espacial para o modelo exponencial e graus moderados de dependência espacial (GD) para os demais ajustes, segundo critério de Cambardella et al. (1994). Os valores dos coeficientes de determinação $\left(\mathrm{R}^{2}\right)$ dos semivariogramas foram maiores que 0,97 para todos os ajustes (Tabela 2), ao passo que as somas de quadrados de desvios ponderados (SQDP) apresentaram maiores valores para o modelo exponencial, seguido dos modelos gaussiano e esférico.

Em seguida, na validação cruzada, foi observado que o ajuste com o modelo esférico apresentou as melhores estatísticas, com coeficiente linear de 0,015; coeficiente angular de 0,999; coeficiente de determinação da validação cruzada $\left(\mathrm{R}^{2}{ }_{\mathrm{vc}}\right)$ de 0,998 ; e erro padrão de estimativa $\left(\mathrm{S}_{\mathrm{yx}} \%\right)$ de $0,51 \%$, sendo o modelo escolhido para confecção do mapa temático por meio da krigagem ordinária. Pela análise semivariográfica foi possível detectar que o volume apresentou estrutura espacial, além de dispersão reduzida dos valores observados juntos da linha média estimada (Figura 1).

De maneira geral foi observado moderado grau de dependência espacial para o volume, segundo critério de Cambardella et al. (1994), sendo possível utilizar a krigagem para gerar mapas. Assim, padrões espaciais distintos foram verificados, nos quais foi notada elevada heterogeneidade espacial do volume (Figura 2), decorrente principalmente da variabilidade espacial da capacidade produtiva.

Ademais, a utilização de valores médios de amostras não é suficiente para caracterizar a variabilidade volumétrica do plantio florestal. Adicionalmente, o povoamento foi dividido em seis estratos volumétricos com intervalo de $30 \mathrm{~m}^{3} / \mathrm{ha}$, variando entre 198,77 e $354,33 \mathrm{~m}^{3} /$ ha. O estrato mais extenso foi o que está na classe $288,77-318,77\left(\mathrm{~m}^{3} / \mathrm{ha}\right)$, gerando um volume total de $1.531 \mathrm{~m}^{3}$. O estrato de menor área está na classe de 198,77-228,77 ( $\left.\mathrm{m}^{3} / \mathrm{ha}\right)$, com um volume total de apenas $42,8 \mathrm{~m}^{3}$. Essas informações permitem distinguir e visualizar características distintas do povoamento, o que possibilita realizar o zoneamento do crescimento das árvores, melhorando a qualidade das informações sobre a quantificação e qualificação das florestas.

Por meio do mapeamento do volume foram observadas delimitações espaciais definindo as classes de produtividade, com áreas aproximadas de

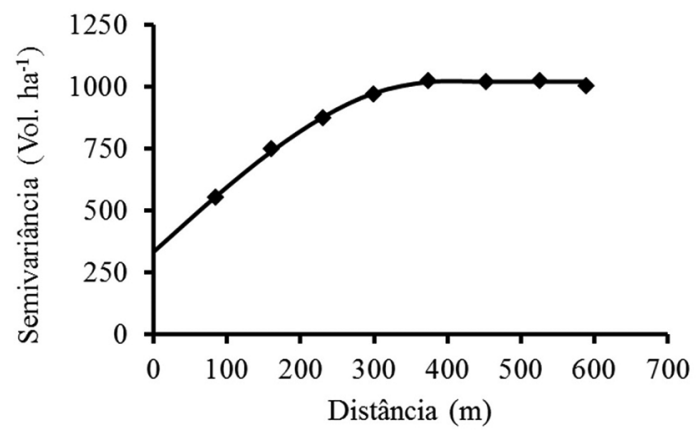

Figura 1. Semivariograma teórico ajustados para o volume por hectare em um povoamentos de Eucalyptus grandis na região de Derrubadas, RS.

Figure 1. Theoretical semivariograms adjusted for the volume per hectare in Eucalyptus grandis in the region of Derrubadas, RS.

Tabela 2. Parâmetros dos modelos geoestatísticos ajustados para o volume por hectare (V) em povoamentos de Eucalyptus grandis na região de Derrubadas, RS.

Table 2. Parameters of geostatistical models adjusted for volume per hectare (V) in Eucalyptus grandis in Derrubadas region, RS.

\begin{tabular}{|c|c|c|c|c|c|c|c|}
\hline Variável & Modelo & $\mathrm{C}_{0}$ & C & $A(m)$ & GD (\%) & $\mathbf{R}^{2}$ & SQDP \\
\hline \multirow{3}{*}{$\begin{array}{c}\mathrm{V} \\
\left(\mathrm{m}^{3} \mathrm{ha}^{-1}\right)\end{array}$} & Esférico & 334,5 & 686,4 & 384,3 & 32,8 & 0,997 & 41,44 \\
\hline & Exponencial & 136,5 & 938,3 & 441,0 & 12,7 & 0,976 & 252,90 \\
\hline & Gaussiano & 460,5 & 565,9 & 338,6 & 44,9 & 0,995 & 94,56 \\
\hline
\end{tabular}

$\mathrm{C}_{\mathrm{o}}=$ efeito pepita; $\mathrm{C}=$ variância a priori (contribuição); $\mathrm{A}=$ alcance; $\mathrm{GD}=$ grau de dependência espacial; $\mathrm{R}^{2}=$ coeficiente de determinação; SQDP = soma de quadrados dos desvios ponderados escalonados. 


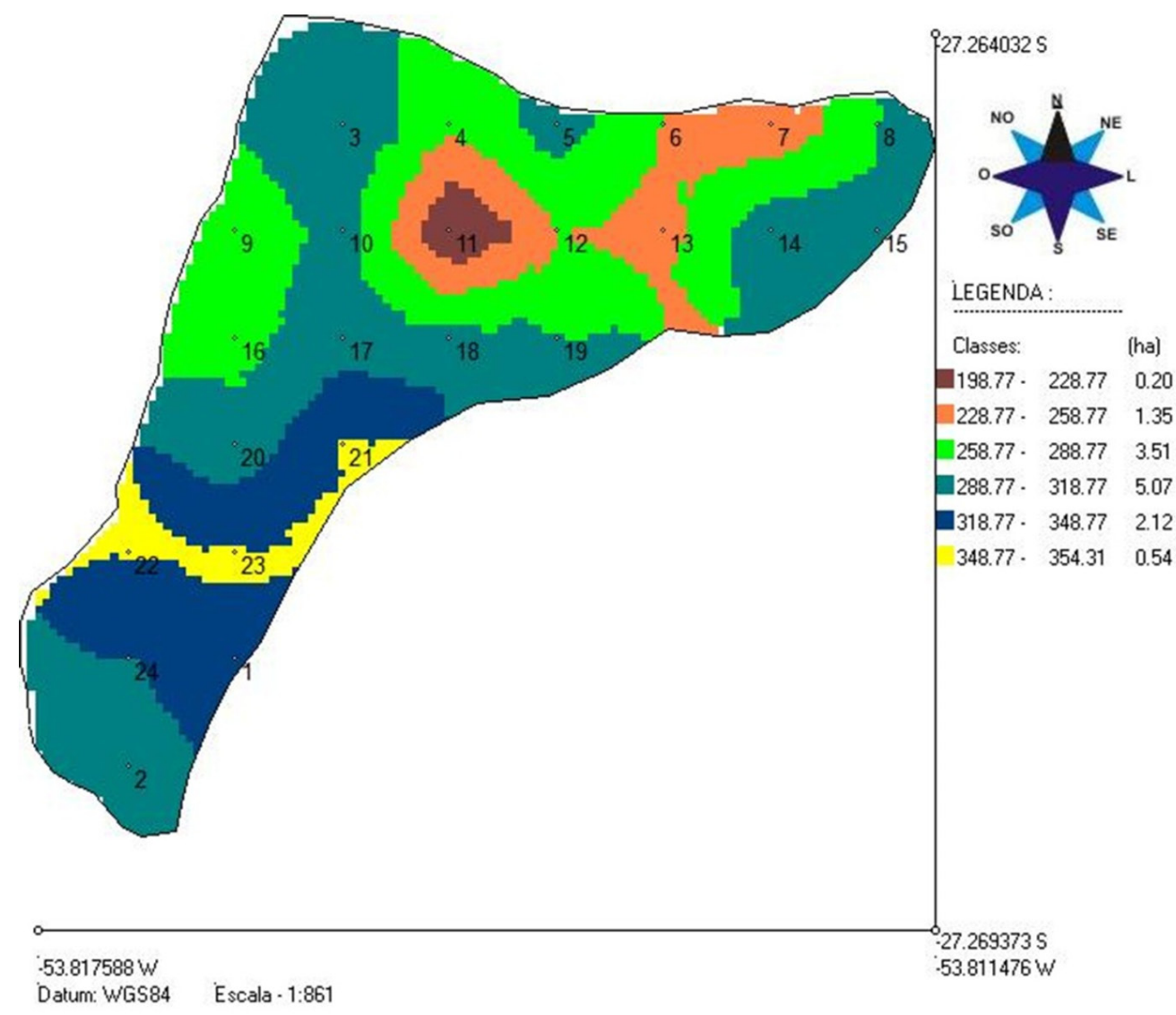

Figura 2. Mapa temático do volume total $\left(\mathrm{m}^{3} \mathrm{ha}^{-1}\right)$ em um povoamento de Eucalyptus grandis na região de Derrubadas, RS.

Figure 2. Thematic map of total volume $\left(\mathrm{m}^{3} \mathrm{ha}^{-1}\right)$ in Eucalyptus grandis in the region of Derrubadas, RS.

1,55; 8,58; e 2,66 ha, para as áreas com menor; média; e alta produtividade, respectivamente. Analisando-se o mapa de krigagem para a variável volume, manejos independentes podem ser aplicados em locais específicos visando regular a densidade do povoamento em benefício do crescimento dos melhores indivíduos.

Para Pelissari et al. (2014), o conhecimento da variabilidade espacial pode ser aplicado à concepção de um sistema de amostragem adequado para as futuras remedições dos povoamentos nos inventários em múltiplas ocasiões, considerando-se a estratificação da floresta em subpopulações homogêneas e a alocação de unidades amostrais em intensidades ideais para a obtenção de estimativas precisas e a custos reduzidos.

A projeção do volume total na área utilizando a geoestatística foi de $3.768,9 \mathrm{~m}^{3}$ (Tabela 3 ), subdividida em seis estratos distintos. Avaliando-se a estimativa do volume total pela amostragem sistemática $\left(3.753,9 \mathrm{~m}^{3}\right)$,
Tabela 3. Classes volumétricas do povoamento, áreas abrangidas por cada classe e volume total com auxílio da análise geoestatística.

Table 3. Stand volumetric classes, areas covered by each class and the total volume with the contribution of geoestatistical analysis.

\begin{tabular}{ccc}
$\begin{array}{c}\text { Classes volumétricas } \\
\left(\mathbf{m}^{\mathbf{3}} \mathbf{h a}\right)\end{array}$ & Área (ha) & Volume total $\left(\mathbf{m}^{3}\right)$ \\
\hline $198,77-228,77$ & 0,20 & 42,8 \\
\hline $228,77-258,77$ & 1,35 & 329,1 \\
\hline $258,77-288,77$ & 3,51 & 971,9 \\
\hline $288,77-318,77$ & 5,07 & 1531,0 \\
\hline $318,77-348,77$ & 2,12 & 704,3 \\
\hline $348,77-354,31$ & 0,54 & 189,8 \\
\hline TOTAL & $\mathbf{1 2 , 7 9}$ & $\mathbf{3 . 7 6 8 , 9}$ \\
\hline
\end{tabular}

percebe-se a diferença do estoque em volume de aproximadamente $15 \mathrm{~m}^{3}$ ou $0,4 \%$. Esse resultado salienta que a krigagem é uma importante alternativa para estimar o estoque de madeira em povoamentos florestais. 
Tabela 4. Volume pós-estratificação do povoamento através do mapa de produtividade.

Table 4. Stand post-stratification volume through productivity map.

\begin{tabular}{|c|c|c|c|c|c|}
\hline Estrato & $\begin{array}{c}\text { Classes de } \\
\text { volume } \\
\text { (m³/ha) }\end{array}$ & N. de parcelas & $\begin{array}{l}\text { Volume médio } \\
\qquad\left(\mathrm{m}^{3} / \mathrm{ha}\right)\end{array}$ & $\begin{array}{c}\text { Área } \\
\text { (ha) }\end{array}$ & $\begin{array}{l}\text { Volume total } \\
\qquad\left(\mathrm{m}^{3}\right)\end{array}$ \\
\hline 1 & $198,77-228,77$ & 1 & 195,7 & 0,20 & 39,1 \\
\hline 2 & $228,77-258,77$ & 4 & 248,3 & 1,35 & 335,2 \\
\hline 3 & $258,77-288,77$ & 3 & 271,6 & 3,51 & 953,2 \\
\hline 4 & $288,77-318,77$ & 12 & 303,7 & 5,07 & $1.539,9$ \\
\hline 5 & $318,77-348,77$ & 1 & 337,2 & 2,12 & 715,0 \\
\hline 6 & $348,77-354,31$ & 3 & 352,8 & 0,54 & 190,5 \\
\hline TOTAL & & & & 12,79 & $3.772,9$ \\
\hline
\end{tabular}

Comparando estimativas pelo estimador clássico e geoestatístico em povoamento de Eucalyptus grandis em São Paulo, Mello et al. (2006) verificaram que o erro amostral do estimador geoestatístico foi menor em $1 \%$ em relação ao estimador clássico, sugerindo o uso do estimador geoestatístico para estimativa por talhão.

\subsection{Inventário tradicional com pós- estratificação}

Com a construção do mapa de produtividade florestal e as dimensões de área de cada estrato realizou-se a pós-estratificação do inventário tradicional alocando-se as unidades amostrais conforme a amplitude entre o limite inferior e superior (Tabela 4). Na análise de variância, o $\mathrm{F}$ calculado $(88,7)$ foi maior que o $\mathrm{F}$ tabelado $(3,16)$, comprovando que existe diferença significativa entre as médias dos estratos, ou seja, a estratificação gerada trará vantagens ao inventário florestal.

Os estratos 2, 3, 4 e 5 foram submetidos à análise estatística pelo processo de amostragem estratificada. A partir do inventário florestal por amostragem estratificada obteve-se um volume total de $3.772,9 \mathrm{~m}^{3}$, com erro padrão da média de $5,84 \mathrm{~m}^{3} / \mathrm{ha}$ e erro relativo de $2,0 \%$. A estimativa do volume total do povoamento com a pós-estratificação do inventário tradicional apresentou resultados muito próximos ao inventário florestal pelo processo de amostragem sistemática (Tabela 1) e a análise geoestatística (Tabela 3).

O erro padrão da média pela amostragem estratificada (2,0\%), quando comparado com o processo de amostragem sistemático $(5,3 \%)$, apresentou uma redução de mais de $160 \%$ para um mesmo tamanho de amostra. Esse resultado salienta a importância da pós-estratificação do volume e permite a redução do erro relativo e aumento da precisão sem causar impactos no custo do inventário.
Resultados semelhantes com redução do erro por meio da krigagem foram encontrados por Guedes et al. (2012) em estudos sobre a possibilidade de uso de interpoladores geoestatísticos para definição de estratos em relação a amostragem casual simples e a amostragem casual estratificada, com redução do erro de amostragem em $47,0 \%$ e $65,7 \%$, respectivamente.

\section{CONCLUSÃO}

O estoque de madeira do povoamento florestal analisado pela amostragem sistemática foi de $3.753,9 \mathrm{~m}^{3}$ e pela krigagem geoestatística, de $3.768,9 \mathrm{~m}^{3}$. Os resultados da análise geoestatística apresentaram estimativa volumétrica de madeira similar ao método tradicional e apresentam grande potencial como forma de pós-estratificação nos inventários florestais, inclusive com substancial redução no erro de amostragem. Além disso, o mapa de produtividade remete a um zoneamento de produção do povoamento florestal a partir do qual pode-se estabelecer critérios de manejo nas etapas de colheita, conforme a produtividade determinada.

\section{STATUS DA SUBMISSÃO}

Recebido: 14 mar., 2014

Aceito: 22 mar., 2016

\section{AUTOR(ES) PARA CORRESPONDÊNCIA}

\section{Marcelo Roveda}

Departamento de Engenharia Florestal, Universidade Federal do Paraná - UFPR, Avenida Prefeito Lothário Meissner, 900, Jardim Botânico, CEP 80210-170, Curitiba, PR, Brasil e-mail: marcelo_roveda@hotmail.com.br 


\section{REFERENNCIAS}

Biondi F, Myers DE, Avery CC. Geostatistically modeling stem size and increment in an old-growth forest. Canadian Journal of Forest Research 1994; 24(1): 1354-1368. http:// dx.doi.org/10.1139/x94-176.

Bognola IA, Justiniano PR Jr, Silva EAA, Lingnau C, Higa AR. Modelagem uni e bivariada da variabilidade espacial de rendimento de Pinus taeda L. Revista Floresta 2008; 38(2): 373-385.

Brandelero C, Antunes MUF, Giotto E. Silvicultura de precisão: mapeamento, inventário e geoestatística. Revista Geomática 2007; 2(1): 15-25.

Cambardella CA, Moorman TB, Novak JM, Parkin TB, Karlen DL, Turco RF et al. Field-scale variability of soil properties in central Iowa soils. Soil Science Society of America Journal 1994; 58(5): 1501-1511. http://dx.doi. org/10.2136/sssaj1994.03615995005800050033x.

Carvalho MP, Mendonça VZ, Pereira FCBL, Arf MV, Kappes C, Dalchiavon FC. Produtividade de madeira do eucalipto correlacionada com atributos do solo visando ao mapeamento de zonas específicas de manejo. Ciência Rural 2012; 42(10): 1797-1803. http://dx.doi.org/10.1590/ S0103-84782012005000078.

Finger CAG. Tabelas para o manejo florestal de Eucalyptus grandis e Eucalyptus saligna, em primeira e segunda rotações. Santa Maria: Universidade Federal de Santa Maria; 1997.

Giotto E. Agricultura de precisão: estruturação de mapas de produtividade a partir de arquivos de produção em formato texto (CSV). Santa Maria: Universidade Federal de Santa Maria; 2008.

Gonçalves JLM, Alvares CA, Gonçalves TD, Moreira RM, Mendes JCT, Gava JL. Mapeamento de solos e da produtividade de plantações de Eucalyptus grandis em Itatinga, SP, com uso de sistema de informação geográfica. Scientia Forestalis 2012; 94(1): 187-201.

Guedes ICL, Mello JM, Mello CR, Oliveira AD, Silva ST, Scolforo JRS. Técnicas Geoestatísticas e Interpoladores espaciais na Estratificação de povoamento de Eucalyptus sp. Ciência Florestal 2012; 22(3): 541-550. http://dx.doi. org/10.5902/198050986621.

Hack C. Respostas da vegetação remanescente e da regeneração natural em uma Floresta Ombrófila Mista cinco anos após intervenções de manejo [dissertação]. Santa Maria: Setor de Ciências Agrárias, Universidade Federal de Santa Maria; 2007.

Höck BK, Payn TW, Shirley JW. Using a geographic information system and geostatistics to estimate site index of Pinus radiata for Kaingaroa Forest, New Zealand. New Zealand Journal of Forestry Science 1993; 23(3): 264-277.

Instituto Brasileiro de Geografia e Estatística - IBGE. Mapas de solos do Brasil. 33rd ed. Rio de Janeiro: IBGE; 1986.
Isaaks EH, Srivastava RM. Applied geoestatistics: an introduction to. New York: Oxford University Press; 1989.

Jesus CM, Miguel EP, Azevedo GB, Azevedo GTOS, Pereira RS. Modelagem hipsométrica em povoamento clonal de Eucalyptus urophylla x Eucalyptus grandis no Distrito Federal. Enciclopédia Biosfera 2015; 11(21): 1298-1308.

Kanegae H Jr, Scolforo JRS, Mello JM, Oliveira AD. Avaliação de interpoladores estatísticos e determinísticos como instrumento de estratificação de povoamentos clonais de Eucalyptus sp. Cerne 2006; 12(2): 123-136.

Leal FA, Miguel EP, Matricardi EAT. Mapeamento de unidades produtivas utilizando a interpolação geoespacial krigagem a partir do inventário florestal em um povoamento de Eucalyptus urophylla S. T. Blake. Enciclopédia da Biosfera 2011; 7(13): 727-745.

Lima CGR, Carvalho MP, Narimatsu KCP, Silva MG, Queiroz HA. Atributos físico-químicos de um Latossolo do cerrado brasileiro e sua relação com características dendrométricas do eucalipto. Revista Brasileira de Ciencia do Solo 2010; 34(1): 163-173. http://dx.doi.org/10.1590/ S0100-06832010000100017.

Marchiori JNC, Sobral M. Dendrologia das angiospermas: Myrtales. Santa Maria: UFSM; 1997.

Mello JM, Batista JLF, Oliveira MS, Justiniano PR Jr. Estudo da dependência espacial de características dendrométricas para Eucalyptus grandis. Cerne 2005; 11(2): 113-126.

Mello JM, Diniz FS, Oliveira AD, Scolforo JRS, Acerbi FW Jr, Thiersch CR. Métodos de amostragem geoestatística para estimativa do número de fustes e volume em plantios de Eucalyptus grandis. Revista Floresta 2009; 39(1): 157-166.

Mello JM, Oliveira MS, Batista JLF, Justiniano PR Jr, Kanegae H Jr. Uso do estimador geoestatístico para predição volumétrica por talhão. Floresta 2006; 36(2): 251-260. http://dx.doi.org/10.5380/rf.v36i2.6454.

Moreno JA. Clima do Rio Grande do Sul. Porto Alegre: Secretaria da Agricultura; 1961.

Nanos N, Tadesse W, Montero G, Gil L, Alia R. Spatial stochastic modeling of resin yield from pine stands. Canadian Journal of Forest Research 2001; 31(1): 1140 1147. http://dx.doi.org/10.1139/x01-047.

Pelissari AL, Figueiredo A Fo, Caldeira SF, Machado SA. Geoestatística aplicada ao manejo de povoamentos florestais de teca, em períodos pré-desbaste seletivo, no estado do Mato Grosso. Revista Brasileira de Biometria 2014; 32(3): 430-444.

Péllico NS, Brena DA. Inventário florestal. Curitiba: Universidade Federal do Paraná; 1997.

Rosa G Fo, Carvalho MP, Montanari R, Silva JM, Siqueira GM, Zambianco EC. Variabilidade espacial de propriedades dendrométricas do eucalipto e de atributos físicos de um Latossolo Vermelho. Bragantia 2011; 70(2): 439-446. http://dx.doi.org/10.1590/S0006-87052011000200027. 
Rufino TMC, Thiersch CR, Ferreira SO, Kanegae H Jr, Fais D. Uso da geoestatística no estudo da relação entre variáveis dentrométricas de povoamentos de Eucalyptus sp. e atributos do solo. Ambiência 2006; 2(1): 83-93.

Santos PC, Santana AC, Barros PLC, Queiroz JCB, Vieira TO. O emprego da geoestatística na determinação do tamanho "ótimo" de amostras aleatórias com vistas à obtenção de estimativas dos volumes dos fustes de espécies florestais em Paragominas, estado do Pará. Acta Amazonica 2011; 41(2): 213-222. http://dx.doi.org/10.1590/ S0044-59672011000200005.

Sousa GTO, Azevedo GB, Barreto PAB, Conceição V Jr. Relações hipsométricas para Eucalyptus urophylla conduzidos sob regime de alto fuste e talhadia no Sudoeste da Bahia. Scientia Plena 2013; 9(4): 1-7.

Trangmar BB, Yost RS, Uehara G. Application of geostatistics to spatial studies of soil properties. Advances in Agronomy 1985; 38(1): 54-94.

Vettorazzi CA, Ferraz SFB. Silvicultura de precisão: uma nova perspectiva para o gerenciamento de atividades florestais. In: Borém A, Giúdice MP, Queiroz DM. Agricultura de precisão. Viçosa: Editora UFV; 2000.

Vieira SR. Uso de geoestatística em estudos de variabilidade espacial de propriedades do solo. In: Novais RF, Alvarez VH, Schaefer, CEGR, organizadores. Tópicos em ciência do solo. Viçosa: Sociedade Brasileira de Ciência do Solo; 2000. 